\title{
Numerical exploration of new features on three- dimensional transition of a cylinder wake
}

\author{
LING Guocan ${ }^{1}$, XIONG Jun ${ }^{1,2}$ \& ZHU Keqin ${ }^{2}$ \\ 1. State Key Laboratory of Nonlinear Mechanics, Institute of Mechanics, Chinese Academy of Sciences, \\ Beijing 100080, China; \\ 2. Department of Engineering Mechanics, Tsinghua University, Beijing 100084, China \\ Correspondence should be addressed to Ling Guocan (email: linggc@imech.ac.cn) \\ Received November 4, 2003
}

\begin{abstract}
The three-dimensional transition of the wake flow behind a circular cylinder is studied in detail by direct numerical simulations using 3D incompressible N-S equations for Reynolds number ranging from 200 to 300 . New features and vortex dynamics of the $3 \mathrm{D}$ transition of the wake are found and investigated. At $R e=200$, the flow pattern is characterized by mode A instability. However, the spanwise characteristic length of the cylinder determines the transition features. Particularly for the specific spanwise characteristic length linear stable mode may dominate the wake in place of mode $A$ and determine the spanwise phase difference of the primary vortices shedding. At $R e=250$ and 300 it is found that the streamwise vortices evolve into a new type of mode — "dual vortex pair mode" downstream. The streamwise vortex structures switch among mode A, mode B and dual vortex pair mode from near wake to downstream wake. At $R e=250$, an independent low frequency $f_{\mathrm{m}}$ in addition to the vortex shedding frequency $f_{\mathrm{s}}$ is identified. Frequency coupling between $f_{\mathrm{m}}$ and $f_{\mathrm{s}}$ occurs. These result in the irregularity of the temporal signals and become a key feature in the transition of the wake. Based on the formation analysis of the streamwise vorticity in the vicinity of cylinder, it is suggested that mode $A$ is caused by the emergence of the spanwise velocity due to three dimensionality of the incoming flow past the cylinder. Energy distribution on various wave numbers and the frequency variation in the wake are also described.
\end{abstract}

Keywords: cylinder, wake, 3D transition, Fourier-spectral-spectral-element method, direct numerical simulation (DNS).

DOI: 10.1360/04za0006

The flow past a circular cylinder is one of the focuses of the investigations in fluid mechanics because of its theoretical significance and applied background. Since the $1980 \mathrm{~s}$ 3D transition of the wake and the transition to the turbulence have received great attention. Generally speaking, with the increase of the Reynolds number the wake is involved into successive instabilities, i.e. the transition from the steady flow to the formation of Kármán vortices, then to 3D streamwise vortex structures, the development of separated shear layer instability, the boundary layer instability, and eventually the wake becomes 
turbulent ${ }^{[1]}$. The extensive studies with both experiments and numerical simulations in the low and moderate Reynolds number regime have revealed many interesting phenomena, such as the discontinuity in the curve of vortex shedding frequency ${ }^{[2]}$, the emergence of mode $\mathrm{A}$ and mode $\mathrm{B}^{[2-5]}$, and the existence of the spot-like large scale structures ${ }^{[6,7]}$. However, understanding of $3 \mathrm{D}$ vortex dynamics during the transition is far from the completion. In the previous works much information provided is about near wake vortex structures. We only have a little knowledge on the flow pattern evolution and the dynamics downstream. Some important problems concerning the physical mechanisms behind the transition phenomena remain unclear, for instance, the effect of the flow conditions on the vortex modes, the origins of modes $\mathrm{A}$ and $\mathrm{B}$, and the vortex dislocations, as well as the route by which the wake is led to the turbulence.

This paper further investigated the characteristics of 3D transition in the regime $200 \leqslant$ $R e \leqslant 300$ by direct numerical simulations. We explored the existence of new transition features based on DNS results and analyzed the vortex dynamics in detail. Furthermore we attempt to explain the origin of 3D vortex shedding mode.

\section{Numerical formulations}

The right-handed Cartesian coordinate system has been established, in which the positive direction of the $x$ axis is the direction of incoming flow and the $z$ axis is the axis of the cylinder. The 3-D N-S and the continuity equations governing the incompressible viscous flow are taken as follows, where all variables are normalized by the diameter of the cylinder $D$ and the uniform stream velocity $U$ :

$$
\begin{gathered}
\partial \boldsymbol{V} / \partial t+\boldsymbol{N}(\boldsymbol{V})=-\nabla p+\nabla^{2} \boldsymbol{V} / R e \\
\nabla \cdot \boldsymbol{V}=0
\end{gathered}
$$

where the Reynolds number is defined as $R e=U D / \nu$, in which $\nu$ is the kinematic viscosity, and the nonlinear convection operator $\boldsymbol{N}(\boldsymbol{V})=\boldsymbol{V} \cdot \nabla \boldsymbol{V}$. The spatial discretization of the N-S equations in the $z$ axis direction employs the Fourier spectral method. The velocity $\boldsymbol{V}$ and the pressure $p$ are expanded into the form of Fourier series

$$
\begin{aligned}
& \boldsymbol{V}(x, y, z, t)=\sum_{k=-M / 2+1}^{M / 2} \boldsymbol{V}_{k}(x, y, t) e^{i \beta k z}, \\
& p(x, y, z, t)=\sum_{k=-M / 2+1}^{M / 2} p_{k}(x, y, t) e^{i \beta k z},
\end{aligned}
$$

where $M$ is the number of Fourier modes, $\beta=2 \pi / L$, in which $L$ denotes the spanwise characteristic length, i.e. spanwise periodic length. Consequently the N-S and the continuity equations are transformed into the forms below,

$$
\begin{gathered}
\partial \boldsymbol{V}_{k} / \partial t+\boldsymbol{F}_{k}[\boldsymbol{N}(\boldsymbol{V})]=-\nabla p_{k}+\left(\nabla_{x y}^{2}-k^{2} \beta^{2}\right) \boldsymbol{V}_{k} / R e \\
\nabla \cdot \boldsymbol{V}_{k}=0
\end{gathered}
$$

Copyright by Science in China Press 2004 
where the forms of the operators $\nabla_{x y}$ and $\nabla$ are respectively

$$
\nabla_{x y}=\partial / \partial x \boldsymbol{e}_{x}+\partial / \partial y \boldsymbol{e}_{y}, \quad \nabla=\nabla_{x y}+i k \boldsymbol{e}_{z} .
$$

$\boldsymbol{F}_{k}[\boldsymbol{N}(\boldsymbol{V})]$ in eq. (4) is the $k$ th coefficient of the Fourier transform of $\boldsymbol{N}(\boldsymbol{V})$. The time discretization of eqs. (4) and (5) employs a high-order splitting algorithm based on the mixed stiffly stable scheme ${ }^{[8]}$. The solving procedure is split into the substeps below

$$
\begin{gathered}
\left(\boldsymbol{V}_{k}^{n+1 / 3}-\sum_{q=0}^{Q-1} \alpha_{q} \boldsymbol{V}_{k}^{n-q}\right) / \Delta t=-\sum_{q=0}^{Q-1} \beta_{q} \boldsymbol{F}_{k}\left[\boldsymbol{N}\left(\boldsymbol{V}^{n-q}\right)\right], \\
\left(\nabla_{x y}^{2}-k^{2} \beta^{2}\right) p_{k}^{n+1}=\nabla \cdot \boldsymbol{V}_{k}^{n+1 / 3} / \Delta t \\
\left(\boldsymbol{V}_{k}^{n+2 / 3}-\boldsymbol{V}_{k}^{n+1 / 3}\right) / \Delta t=-\nabla p_{k}^{n+1}, \\
\left(\gamma_{0} \boldsymbol{V}_{k}^{n+1}-\boldsymbol{V}_{k}^{n+2 / 3}\right) / \Delta t=\left(\nabla_{x y}^{2}-k^{2} \beta^{2}\right) \boldsymbol{V}_{k}^{n+1} / R e,
\end{gathered}
$$

where $\boldsymbol{V}_{k}^{n+1 / 3}$ and $\boldsymbol{V}_{k}^{n+2 / 3}$ are intermediate values of the velocities, $\alpha_{q}$ and $\beta_{q}$ are implicit/explicit weight coefficients for the stiffly stable scheme of order $Q$, and $\gamma_{0}$ is the weight coefficient of the backwards differentiation scheme. Their values for $Q=3$, which is employed in this paper, are listed in table 1. Eqs. (7) and (9) are explicitly solved for $\boldsymbol{V}_{k}^{n+1 / 3}$ and $\boldsymbol{V}_{k}^{n+2 / 3}$ respectively; while eqs. (8) and (10), which are in the form of Helmholtz equations for $p_{k}$ and $\boldsymbol{V}_{k}^{n+1}$ respectively, are solved by the spectral element $\operatorname{method}^{[9]}$.

Table 1 Values of weight coefficients of the mixed stiffly stable scheme for $Q=3$

\begin{tabular}{rcccccc}
\hline$\gamma_{0}$ & $\alpha_{0}$ & $\alpha_{1}$ & $\alpha_{2}$ & $\beta_{0}$ & $\beta_{1}$ & $\beta_{2}$ \\
\hline $11 / 6$ & 3 & $-3 / 2$ & $1 / 3$ & 3 & -3 & 1 \\
\hline
\end{tabular}

For the problem of the flow past a cylinder the velocity is zero on the cylinder surface, and unity on the inlet surface. The normal derivative of the velocities is zero on the other boundaries. On the outlet surface $p=0$, and on the other boundaries

$$
\partial p_{k}^{n+1} / \partial n=-\boldsymbol{n} \cdot\left\{\sum_{q=0}^{Q-1} \beta_{q} \boldsymbol{F}_{k}\left[\boldsymbol{N}\left(\mathbf{V}^{n-q}\right)\right]+1 / R e \sum_{q=0}^{Q-1} \beta_{q} \nabla \times\left(\nabla \times \boldsymbol{V}_{k}^{n-q}\right)\right\}
$$

where $\boldsymbol{n}$ denotes the unit vector normal to the computational domain boundary. The form of the initial condition for the 3D computations is the corresponding periodic 2D flow superposed by the spatial random disturbance of $\boldsymbol{V}$, the maximum amplitude of which is $1.0 \times 10^{-4}$.

\section{Numerical results}

Before performing the 3D numerical simulations of the cylinder wake, the $2 \mathrm{D}$ flow in the regime $50 \leqslant R e \leqslant 300$ is simulated to verify the algorithms and the program codes, as well as to provide the initial conditions for the $3 \mathrm{D}$ simulations.

To assess the effect of the size of the computational domain on the numerical results, the numerical tests use various meshes marked as meshes A, B and C, the size of which 
successively increases. The mesh is illustrated in fig. 1 and the size parameters are listed in table 2. The results of Strouhal number and drag coefficient are shown in figs. 2 and 3 respectively. The results for the maximum mesh, e.g. mesh $\mathrm{C}$, are conformed to that of the previous studies ${ }^{[10-19]}$, especially that of refs. $[10,11]$. However, the present $3 \mathrm{D}$ simulations employ mesh $\mathrm{B}$, the results of which is a little higher than the previous ones due to the block effect of the computational domain. The other mesh parameters of the 3D computations are listed in table 3 . The Strouhal number and drag coefficient obtained from the 3D simulations are also illustrated in figs. 2 and 3. The 3D effect qualitatively conforms to the results of previous studies. Particularly the differences between the results of $3 \mathrm{D}$ and $2 \mathrm{D}$ simulations are quite close to that of ref. [11].

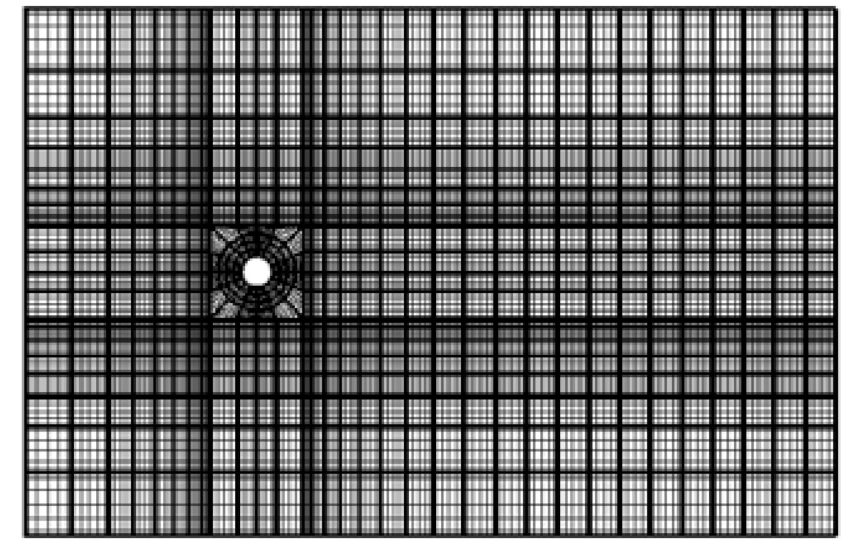

Fig. 1. Mesh in the $x-y$ plane for simulating the flow past a cylinder with the spectral element method. Table 2 Size parameters of the mesh in the $x$ - $y$ plane

\begin{tabular}{ccccccc}
\hline Mesh & $X L_{1}$ & $X L_{2}$ & $Y L$ & $K$ & $N T$ & $N N$ \\
\hline A & 3.5 & 14 & 7 & 34 & 614 & 2754 \\
B & 7.5 & 18.7 & 17 & 688 & 7928 & 33712 \\
C & 16.5 & 36.2 & 33 & 1544 & 17500 & 75656 \\
\hline
\end{tabular}

Note: $X L_{1}, X L_{2}$ are the distances from the inlet and outlet boundaries to the center of the cylinder respectively. $Y L$ is the distance between the upper and lower boundaries of the computational domain. $K$ is the number of the elements. $N T$ is the number of the exterior nodes. $N N$ is the number of nodes, including exterior ones and interior ones.

The detailed study of the flow at $R e=200$ can be found in our previous papers ${ }^{[20,21]}$, so only a brief introduction is given here. $R e=200$ is just beyond the critical Reynolds number of mode $\mathrm{A}$. The near wake is in 3D quasi-periodic laminar state and present 3D transitional behaviors, such as mode A vortex shedding, which is in agreement with previous studies. However, it has been found that the spanwise characteristic length of the cylinder determines the wave length of the streamwise vortex loops, as well as the global properties of the wake. Particularly for the specific spanwise characteristic length linear stable mode, e.g. mode $\lambda / D=6$ for $L / D=12$, can dominate the wake in place of mode $\mathrm{A}$ and determine the spanwise phase difference of the primary vortices shedding. It indicates that the behavior of a dynamical system is influenced by the flow conditions. 


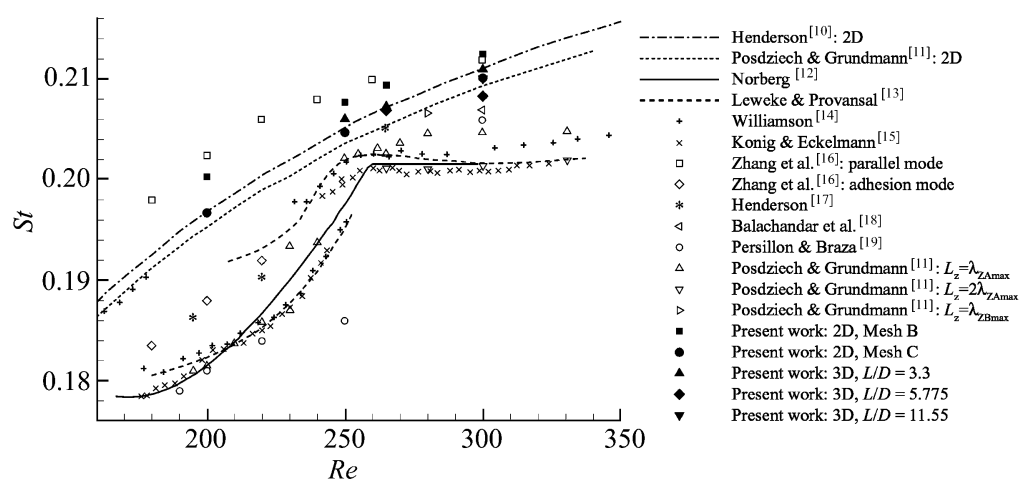

Fig. 2. Strouhal number of the flow past a cylinder.

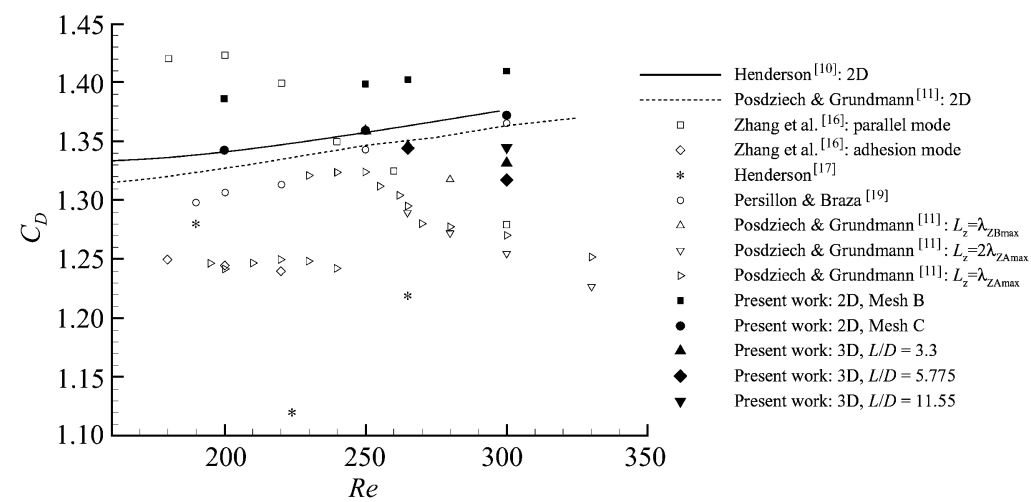

Fig. 3. Drag coefficient of the flow past a cylinder.

Table 3 Parameters for the 3D simulations

\begin{tabular}{cccccc}
\hline \multirow{2}{*}{$L$} & $M$ & \multicolumn{3}{c}{ Mode A } & \multicolumn{3}{c}{ Mode B } \\
\cline { 3 - 6 } & 24 & 1 & 3.3 & 4 & $\lambda$ \\
\hline 3.3 & \multirow{2}{*}{3.775} & 1 & 5.775 & 7 & \\
& & 2 & 2.8875 & & \\
& \multirow{2}{*}{11.55} & 2 & 5.775 & & \\
& 36 & 3 & 3.85 & 14 & \\
& & 4 & 2.8875 & & \\
\hline
\end{tabular}

Now let us put the emphasis on the transition features and vortex dynamics at $R e=$ 250 , where the key feature is dominated by the interaction between modes A and B. In the the present investigations the time signals of lift/drag coefficients are pseudo-periodic. So a "local period" is defined as the time interval between the adjacent minimum points in spanwise-averaged lift force curve. Consequently the "local" Strouhal number and lift/drag coefficients are calculated so as to investigate the temporal evolution of the wake. At $R e=250$ mode $\mathrm{A}$ is unstable, but mode $\mathrm{B}$ is subcritical. The evolution process of the local vortex shedding frequency can be divided into three stages: stage of linear growth of disturbance when $t<100$, transient stage when $100<t<250$, and equilibrium stage 
of the pseudo-periodic state when $t>250$, as shown in fig. 4(a). It actually corresponds to the evolution of the flow pattern that mode A spontaneously emerges in the wake preceding all the other spanwise modes. Then it excites the linear stable mode B through the nonlinear interactions among the various spanwise modes. Eventually multiple modes coexist in the wake, which conforms to the previous studies ${ }^{[17,22]}$ and also validates the algorithm and the program codes employed.
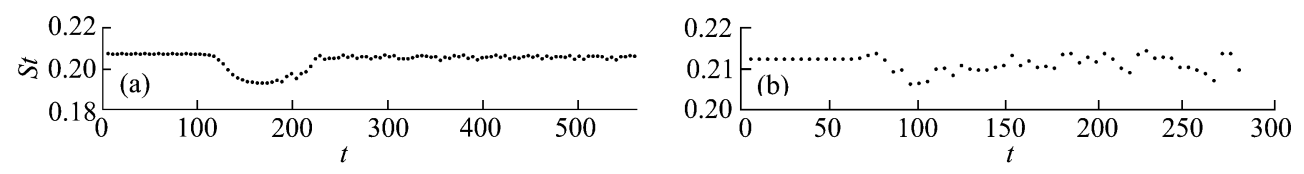

Fig. 4. Local vortex shedding frequency. (a) $R e=250$; (b) $R e=300$.

The streamwise vortex structures in different stages at $R e=250$ are shown in fig. 5. At $t=162$, when the wake is in the transient stage, the streamwise vortex pairs first emerge in the boundary layer on the rear surface of the cylinder and shed downstream. Their spanwise wavelength, which is $3.3 D$ and remains unchanged in the wake, and the anti-phase property of the streamwise vortices of the adjacent vortex braids indicate that it is the mode of typical mode A vortex shedding. The flow pattern is shown in fig. 5(a). At $t=335.1$, when the wake is in the equilibrium stage, the wavelength of the streamwise vortex pairs is still $3.3 D$ near the rear surface of the cylinder, but it changes downstream. In the vortex formation region the wavelength of the vortex pairs becomes $0.825 D$, which is typically mode B. Downstream the spanwise wavelength of the streamwise vortex column restore to $3.3 D$, which is the same as mode $\mathrm{A}$, but the topological structure of the streamwise vortices has greatly changed. The phase (also the sign) of the streamwise vortices is arranged alternately two-positive by two-negative in spanwise direction, which is in opposite to the one-positive-by-one-negative arrangement of mode A. Actually it is a new type of 3D vortex shedding mode, which is never addressed by the previous studies. So it is named as " dual vortex pair mode" here. From the surface of the cylinder to the formation region of the vortices and then to the wake downstream, the structure of the streamwise vortices change from mode A to mode B and then to dual vortex pair mode, as shown in fig. $5(\mathrm{~b})$.
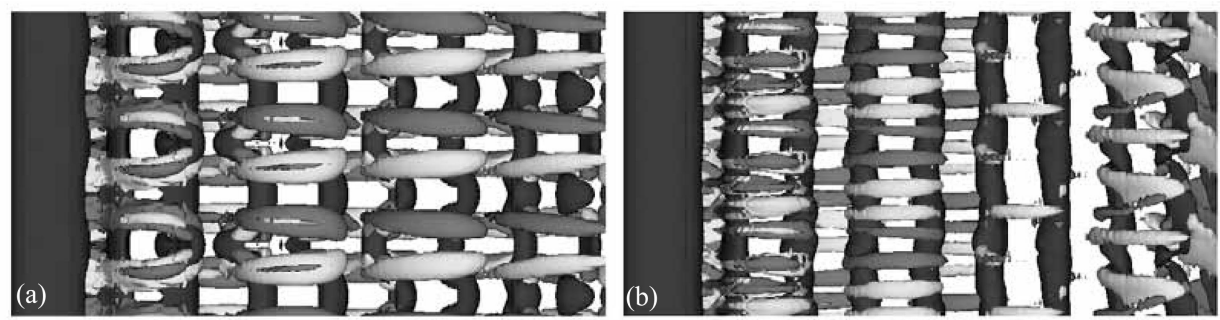

Fig. 5. The spatial evolution of the streamwise vortex structures at $R e=250$. (a) $t=162$, mode A; (b) $t=335.1$. Along flow direction the flow pattern changed from mode A to mode B and then to dual vortex pair mode. (In this figure the spanwise length of the cylinder is extended from $3.3 D$ to $9.9 D$ ). 
The kinetic energy of the various Fourier modes (on wavenumber $k$ ) is calculated by

$$
\begin{aligned}
& E_{k}(t ; x, y)=\left(\left|u_{k}\right|^{2}+\left|v_{k}\right|^{2}+\left|w_{k}\right|^{2}\right) / 2, \\
& k=0,1,2, \cdots, M / 2-1, \quad t>0 .
\end{aligned}
$$

The distribution of time mean energy among the spanwise modes at $R e=250$ at the specific points along the streamwise direction shown in fig. 6 is examined. For the transient stage the time averaging interval is $100 \leqslant t \leqslant 130$ and the result is illustrated in fig. 7(a). The energy distributed on higher wavenumbers is observed, however, it decreases with wavenumber $k$ for each point. Thus mode $k=1$ contains the maximum energy among the spanwise modes except for mode $k=0$, which corresponds the energy held in a uniform flow and consequently represents no spanwise flow patterns. Note that the wavelength of mode $k=1$ corresponds mode A. So the wake in the transient stage at $R e=250$ is characterized by mode $\mathrm{A}$, which is consistent with the flow pattern of the spanwise vortex isosurface illustrated in fig. 5(a).

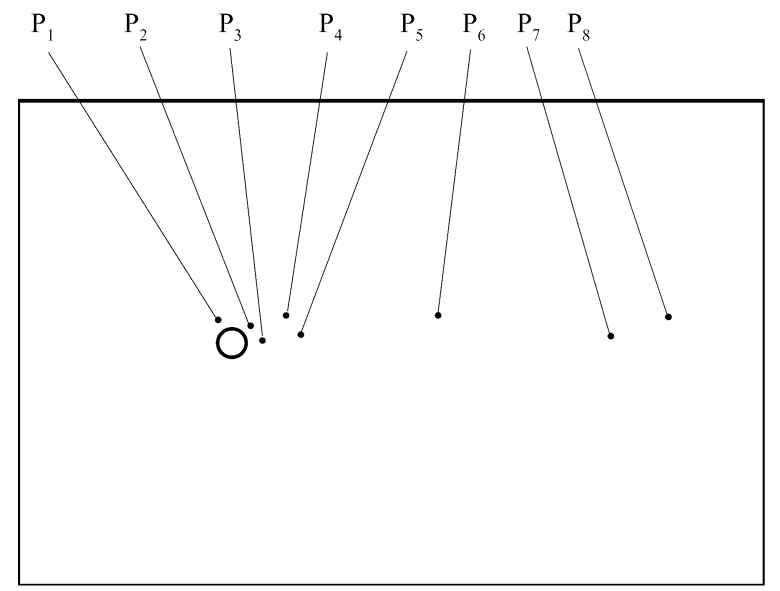

Fig. 6. Positions in the domain of computation where the time signals are recorded in the simulations for the energy analysis.
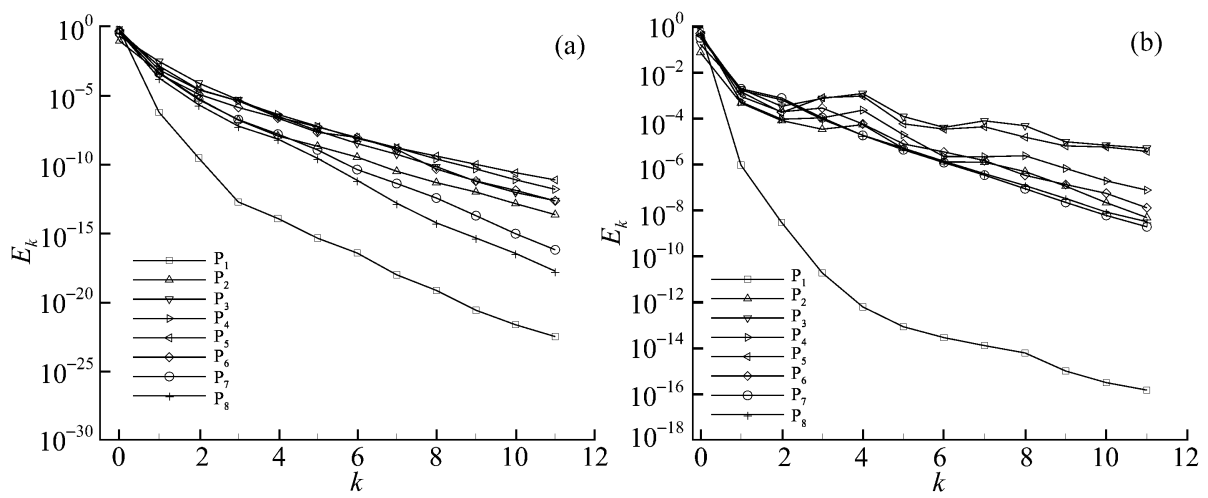

Fig. 7. Mean kinetic energy distribution at the points shown in fig. $6, R e=250$. (a) In the transient stage, $100 \leqslant t \leqslant 130 ;$ (b) in the equilibrium stage, $250 \leqslant t \leqslant 550$. 
As time increases, in the equilibrium stage the energy on the spanwise modes at point $\mathrm{P}_{1}$ is relatively low due to its upstream position. At points $\mathrm{P}_{2}-\mathrm{P}_{5}$ there exists a local maximum at $k=4$ in the energy curves besides the high energy of mode $k=1$. Note that the wavelengths of modes $k=1$ and $k=4$ correspond modes $\mathrm{A}$ and $\mathrm{B}$ respectively. Thus it indicates that modes $\mathrm{A}$ and $\mathrm{B}$ coexist in the near wake. At points $\mathrm{P}_{6}-\mathrm{P}_{8}$ downstream the maxima at mode $k=4$ disappears and mode $k=1$ is dominant. Note that the spanwise wavelength of the dual vortex pair mode is identical to that of mode A, so the energy curves cannot distinguish one from another. The streamwise vortex isosurfaces in fig. 5(b) indicate that mode $k=1$ corresponds not to mode A but the dual vortex pair mode in the far wake.

The coexistence of multiple modes results in the complexity of the spectrum of the time signals of the key quantities in the wake. The spectra of the drag coefficient in different stages are illustrated in fig. 8. In the transient stage the spectrum is quite simple, the energy concentrates upon the vortex shedding frequency $f_{\mathrm{s}}=0.2067$ and its harmonic components. While in the equilibrium stage there exists a pronounced independent low frequency at $f_{\mathrm{m}}=0.0467$. The interaction between $f_{\mathrm{m}}$ and $f_{\mathrm{s}}$ generates coupling frequencies $2 f_{\mathrm{s}}-f_{\mathrm{m}}, 2 f_{\mathrm{s}}+f_{\mathrm{m}}$, and so on. The comparison between fig. $8(\mathrm{a})$ and (b) suggests that the emergence of $f_{\mathrm{m}}$ is due to the coexistence of multiple modes in the wake. The time signals of various spanwise components of the drag coefficient shown in fig. 9 reveal the close connection between $f_{\mathrm{m}}$ and the $3 \mathrm{D}$ vortex shedding modes further. When $t>250$, the time signal of mode $k=1$ has a distinct period of about 20 (note $1 / f_{\mathrm{m}}=21.43$ ) other than the vortex shedding frequency; while the other modes are irregular. It suggests the effect of mode A on $f_{\mathrm{m}}$. Considering the fact that $f_{\mathrm{m}}$ does not emerge in the transient stage, when mode A dominates the wake, it is reasonably inferred that $f_{\mathrm{m}}$ derives itself from mode $\mathrm{A}$ under the influence of mode $\mathrm{B}$.
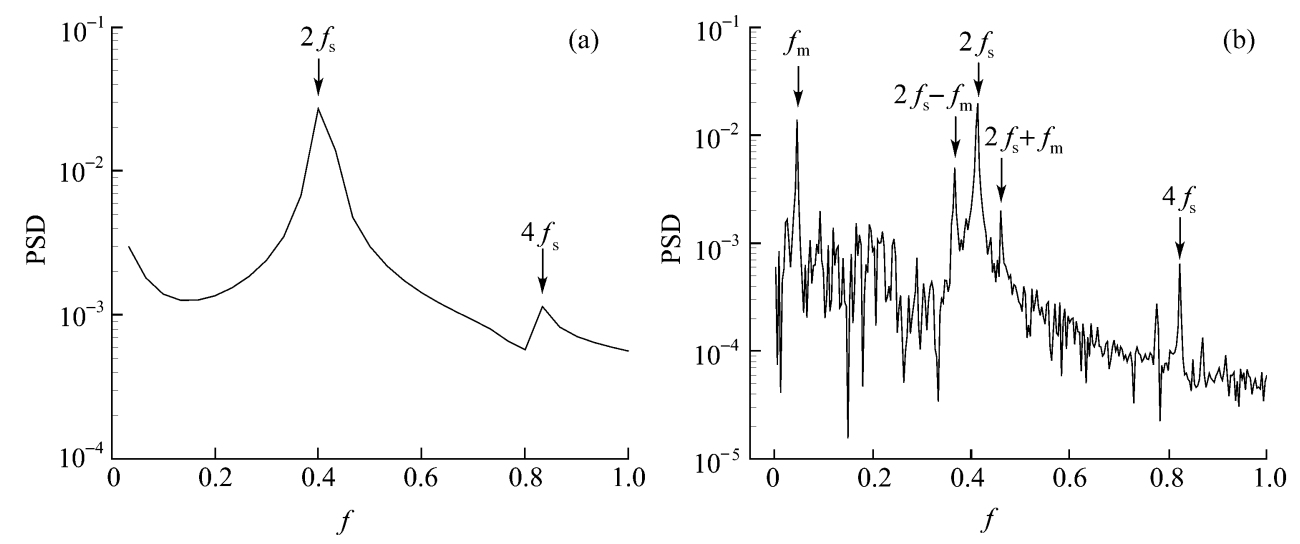

Fig. 8. Spectra of the time signals of drag coefficient, $R e=250, z=0$. (a) $100 \leqslant t \leqslant 130$; (b) $250 \leqslant t \leqslant 550$

Henderson $^{[17]}$ suggested that three dimensionality in the wake leads to the irregular states of the flow and fast transition to turbulence by Ruelle-Takens-Newhouse (RTN) 
route at Reynolds numbers just beyond the onset of the secondary instability. A key feature is the competition between self-excited 3D instability modes in the mode A wavelength band. While in the present simulations, there exists only one mode in the mode A band, and the interaction between $f_{\mathrm{m}}$ and $f_{\mathrm{s}}$ generates coupling frequencies so that the irregularity of the wake considerably increases. It is speculated to be another important mechanism of transition to spatio-temporal chaos and even turbulence in the wake.
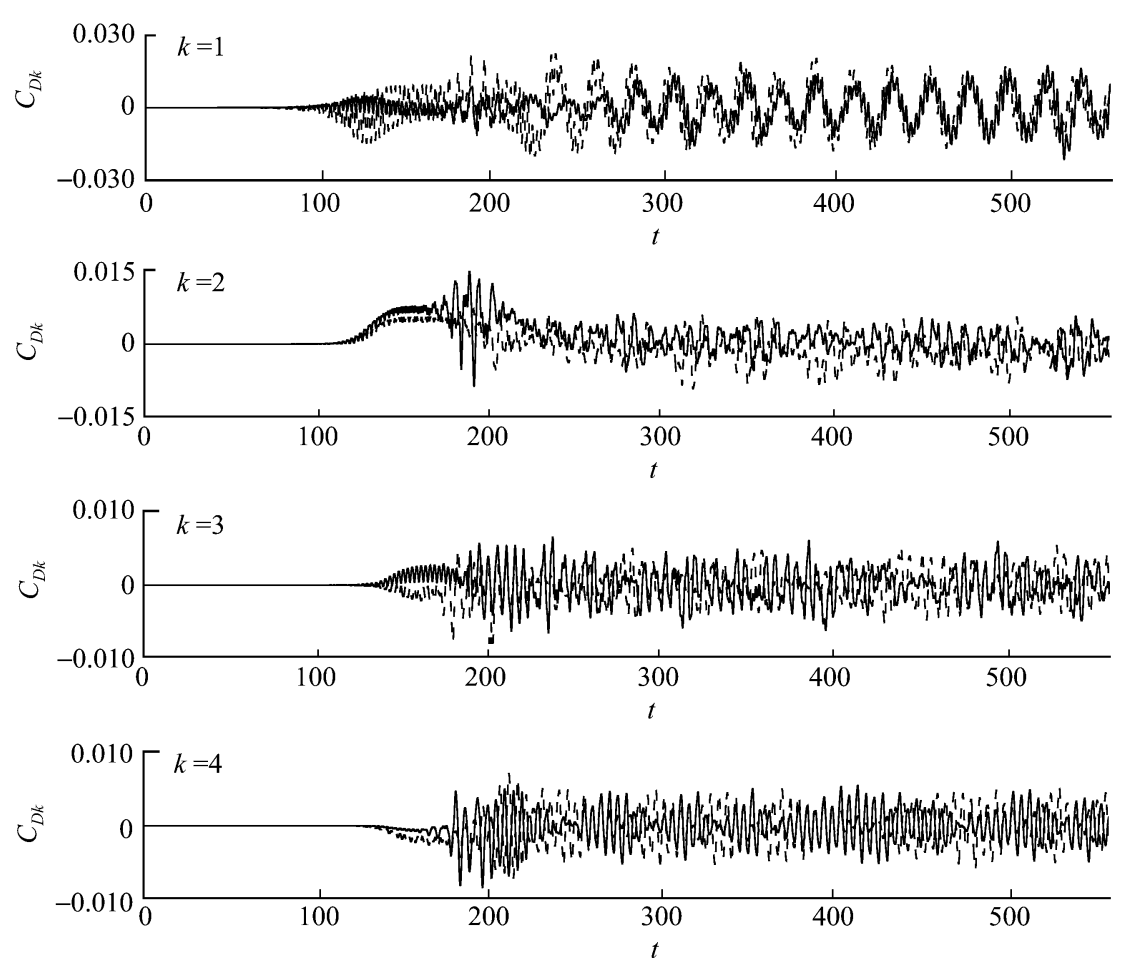

Fig. 9. The time signals of various spanwise components of the drag coefficient, $R e=250$. The solid and dashed lines denote the real and imaginary parts of the Fourier mode respectively.

The physical mechanism of the onset of 3D instability is still under debate. Concerning mode A, Williamson ${ }^{[3]}$ suggested that it is caused by the elliptical instability of the vortex cores during the shedding of the Kármán vortices; Zhang et al. ${ }^{[16]}$ and Brede et al. ${ }^{[4]}$ suggested that it is due to the centrifugal instability of the vortex braid; Braza et al. ${ }^{[7]}$ suggested that it results from the three dimensionality of the incoming flow when passing the cylinder. In the present study streamwise vortex structures of mode A are always located on the rear surface of the cylinder in both transient and equilibrium stages at various Reynolds numbers. So the investigation on its composition may help to determine the origin of mode A.

The expression $\omega_{x}=\partial w / \partial y-\partial v / \partial z$ itself indicates that the streamwise vorticity is composed of two parts, e.g. the transverse gradient of the spanwise velocity and the spanwise gradient of the transverse velocity. The contours of the spanwise velocity $w$ and 
streamwise vorticity $\omega_{x}$ in the plane $x=0$ at $R e=250$ are illustrated in fig. 10 . Despite its tiny order of magnitude, the distribution of $w$, particularly the spanwise wavelength, is identical with that of $\omega_{x}$. The distributions of $\omega_{x}$ and its two compositions in the direction of $y$-axis shown in fig. 11 are quantitative further results. $\omega_{x}$ concentrates in the region within $0.1 D$ apart from the cylinder. And the curves of $\partial w / \partial y$ and $\omega_{x}$ almost coincide with each other in the vicinity of the cylinder. Consequently the streamwise vorticities are mainly composed of the transverse gradient of the spanwise velocity near the cylinder. Since the emergence of the spanwise velocity is the result of the three dimensionality of the incoming flow when passing the cylinder, it is reasonably speculated that the onset
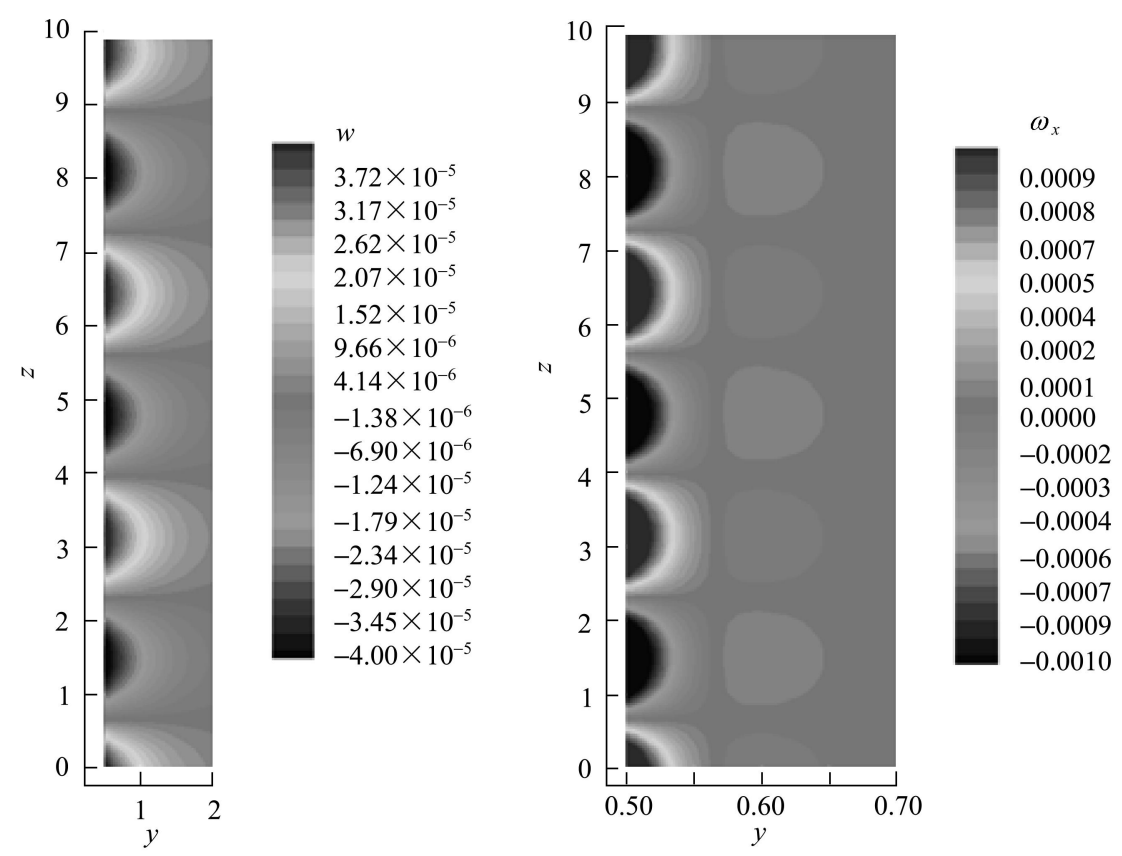

Fig. 10. Contours of the spanwise velocity and streamwise vorticity in the plane $x=0, R e=250, t=56.4$.

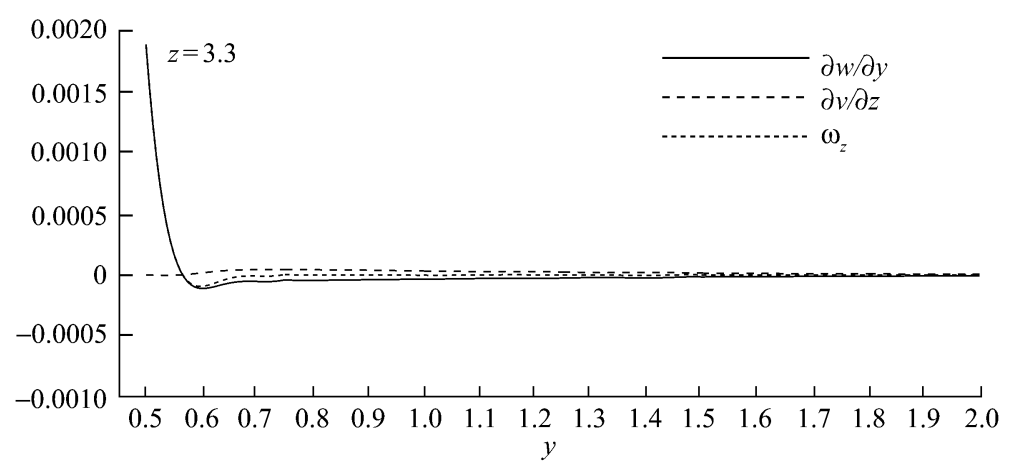

Fig. 11. Distribution of the spanwise velocity and streamwise vorticity in the direction of $y$-axis on the line $x=0, z=3.3, R e=250, t=56.4$.

Copyright by Science in China Press 2004 
of mode $\mathrm{A}$ is the result of the three dimensionality when the incoming flow passes the cylinder rather than due to the instability of the Kármán vortices.

At $R e=300$, modes A and B are both linearly unstable. Compared with that of $R e=250$, the local vortex shedding frequency decreases and becomes pseudo-periodic. It varies with time with much irregularity. By the study on the vortex structures in the wake it is clear that this process corresponds to different temporal evolution of the 3D vortex structures: Due to its higher growth rate mode B replaces the 2-D wake first. Then with the growth of mode A, the two modes coexist in the wake and excite the dual vortex pair mode downstream like that of $R e=250$. The difference is that the streamwise vortices restore the form of mode A further downstream in the far wake. In addition the energy of spanwise modes with higher wavenumbers increases compared with that of $R e=250$, which indicates the further excitation of the small scale structures.

\section{Conclusion}

The present numerical results confirm further some basic features given by the previous investigations, e.g. the change of the vortex shedding frequency and the lift/drag coefficients with the Reynolds number and with time, and the coexistence of modes A and $B$ in the wake. Besides new features on flow transition and vortex dynamics that have not been noticed before are addressed.

$R e=200$ is just beyond the critical Reynolds number of mode A. The numerical results of the present paper indicate that the near wake at this supercritical Reynolds number presents some transitional behavior. The spanwise characteristic length determines the transition features and global properties of the wake. Particularly for the specific spanwise characteristic length, e.g. mode $\lambda / D=6$ for $L / D=12$, linear stable mode can dominate the wake in place of mode A and determine the spanwise phase difference of the primary vortices shedding.

At $R e=250$ and 300 it is found that the streamwise vortices evolve into a new type of mode__ "dual vortex pair mode" downstream. From the surface of the cylinder to the formation region of the vortices then to the wake downstream, the structure of the streamwise vortices switches among mode A, mode B and dual vortex pair mode. At $R e=250$, an independent low frequency $f_{\mathrm{m}}$ other than the vortex shedding frequency $f_{\mathrm{s}}$ is identified. Interaction between $f_{\mathrm{m}}$ and $f_{\mathrm{s}}$ occurs and leads to spectrum broadening. They result in the irregularity of the temporal signals and may be a key factor of the transition of the wake to the spatio-temporal chaos and turbulence. Based on the formation analysis of the streamwise vorticity near the surface of the cylinder, it is suggested that mode $\mathrm{A}$ is originated in the three-dimensionality of the incoming flow past the cylinder and the emergence of the spanwise velocity component. 
In addition the distribution of energy among the spanwise modes with different wave numbers, and the change of the spectrum are presented.

The new findings of the transition phenomena and vortex dynamics, e.g. the dual vortex pair mode and the independent low frequency $f_{\mathrm{m}}$, are based on the present DNS results. Further experimental researches on these features are expected.

Acknowledgements The present study is completed in the State Key Laboratory of Nonlinear Mechanics, Institute of Mechanics, Chinese Academy of Sciences. This work was supported by the State Key Fundamental Research Project of "Large Scale Scientific Computation Research" (Grant No. G199903281), and partly supported by NNSFC (Grant No. GN10272104), as well as by CAS project. LSSC, Institute of Computational Mathematics, Chinese Academy of Sciences is also acknowledged for offering the computational resources of PC clusters.

\section{References}

1. Williamson, C. H. K., Vortex dynamics in the cylinder wake, Annu. Rev. Fluid Mech., 1996, 28: 477—539.

2. Williamson, C. H. K., The existence of two stages in the transition to three dimensionality of a cylinder wake, Phys. Fluids, 1988, 31(11): 3165-3167.

3. Williamson, C. H. K., Three-dimensional wake transition, J. Fluid Mech., 1996, 328: 345—407.

4. Brede, M., Eckelmann, H., Rockwell, D. et al., On secondary vortices in the cylinder wake, Phys. Fluids, 1996, 8(8): 2117-2124.

5. Barkley, D., Henderson, R. D., Three-dimensional Floquet stability analysis of the wake of a circular cylinder, J. Fluid Mech., 1996, 322: 215-241.

6. Williamson, C. H. K., The natural and forced formation of spot-like dislocations in the transition of a wake, J. Fluid Mech., 1992, 243: 393-441.

7. Braza, M., Faghani, D., Persillon, H., Successive stages and the role of natural vortex dislocations in threedimensional wake transition, J. Fluid Mech., 2001, 439: 1—41.

8. Karniadakis, G. E., Israeli, M., Orszag, S. A., High-order splitting methods for incompressible Navier-Stokes equations, J. Comput. Phys., 1991, 97: 414 443.

9. Xiong, J., Investigation on the 3D Transition Features in the Wake of a Cylinder (in Chinese): [doctor thesis], Beijing: Department of Engineering Mechanics, Tsinghua University, 2003.

10. Henderson, R. D., Details of the drag curve near the onset of vortex shedding, Phys. Fluids, 1995, 7: 21022121.

11. Posdziech, O., Grundmann, R., Numerical simulation of the flow around an infinitely long circular cylinder in the transition regime, Theoret. Comput. Fluid Dynamics, 2001, 15: 121-141.

12. Norberg, C., An experimental investigation of the flow around a circular cylinder: influence of aspect ratio, J. Fluid Mech., 1994, 258: 287-316.

13. Leweke, T., Provansal, M., The flow behind rings: bluff body wakes without end effects, J. Fluid Mech., 1995, 288: $265-310$.

14. Williamson, C. H. K., Oblique and parallel modes of vortex shedding in the wake of a circular cylinder at low Reynolds numbers, J. Fluid Mech., 1989, 206: 579-627.

15. König, M., Eckelmann, H., An experimental study of the three-dimensional structure of the wake of circular cylinders in the laminar and transitional Reynolds number range, in Proceedings of the IUTAM Symposium on Bluff-body Wakes and Instabilities (eds. Eckelmann, H., Graham, J. M. R., Huerre, P. et al.), Berlin: SpringerVerlag, 1993, 341-344.

16. Zhang, H. Q., Fey, U., Noack, B. R. et al., On the transition of the cylinder wake, Phys. Fluids, 1995, 7: 779-794.

17. Henderson, R. D., Nonlinear dynamics and pattern formation in turbulent wake transition, J. Fluid Mech., 1997, 352: $65-112$.

18. Balachandar, S., Mittal. R., Najjar, F. M., Properties of the mean recirculation region in the wakes of twodimensional bluff bodies, J. Fluid Mech., 351: 167-199.

19. Persillon, H., Braza, M., Physical analysis of the transition to turbulence in the wake of a circular cylinder by three-dimensional Navier-Stokes simulation, J. Fluid Mech., 1998, 365: 23-88.

20. Xiong, J., Ling, G. C., Zhu, K. Q., Features of the supercritical transition in the wake of a circular cylinder, Acta Mechanica Sinica (in English), 2002, 18(6): 567-580.

21. Xiong, J., Ling, G. C., Zhu, K. Q., The effects of the spanwise characteristic length on the transition feature in the wake of a circular cylinder, Chinese Journal of Aeronautics, 2003, 16(2): 65-68.

22. Thompson, M., Hourigan, K., Sheridan, J., Three-dimensional instabilities in the wake of a circular cylinder, Exp. Therm. Fluid Sci., 1996, 12(2): 190-196.

Copyright by Science in China Press 2004 